\title{
Emotional and physical health benefits of expressive writing
}

\author{
Karen A. Baikie \& Kay Wilhelm
}

\begin{abstract}
Writing about traumatic, stressful or emotional events has been found to result in improvements in both physical and psychological health, in non-clinical and clinical populations. In the expressive writing paradigm, participants are asked to write about such events for 15-20 minutes on 3-5 occasions. Those who do so generally have significantly better physical and psychological outcomes compared with those who write about neutral topics. Here we present an overview of the expressive writing paradigm, outline populations for which it has been found to be beneficial and discuss possible mechanisms underlying the observed health benefits. In addition, we suggest how expressive writing can be used as a therapeutic tool for survivors of trauma and in psychiatric settings.
\end{abstract}

Over the past 20 years, a growing body of literature has demonstrated the beneficial effects that writing about traumatic or stressful events has on physical and emotional health. In the first study on expressive writing (Pennebaker \& Beall, 1986), college students wrote for 15 minutes on 4 consecutive days about 'the most traumatic or upsetting experiences' of their entire lives, while controls wrote about superficial topics (such as their room or their shoes). Participants who wrote about their deepest thoughts and feelings reported significant benefits in both objectively assessed and self-reported physical health 4 months later, with less frequent visits to the health centre and a trend towards fewer days out of role owing to illness. The authors concluded that:

'writing about earlier traumatic experience was associated with both short-term increases in physiological arousal and long-term decreases in health problems' (Pennebaker \& Beall, 1986: p. 280).

\section{Expressive writing studies}

The basic writing paradigm (Pennebaker, 1994, 1997a, 1997b; Smyth \& Pennebaker, 1999) used in most of the subsequent expressive writing studies involves participants writing about traumatic or emotional experiences (Box 1) for 3-5 sessions, often over consecutive days, for 15-20 minutes per session. Most studies have been conducted in the laboratory,

\section{Box 1 Typical writing instructions}

For the next 4 days, I would like you to write your very deepest thoughts and feelings about the most traumatic experience of your entire life or an extremely important emotional issue that has affected you and your life. In your writing, I'd like you to really let go and explore your deepest emotions and thoughts. You might tie your topic to your relationships with others, including parents, lovers, friends or relatives; to your past, your present or your future; or to who you have been, who you would like to be or who you are now. You may write about the same general issues or experiences on all days of writing or about different topics each day. All of your writing will be completely confidential.

Don't worry about spelling, grammar or sentence structure. The only rule is that once you begin writing, you continue until the time is up.

Karen Baikie is a clinical psychologist and postdoctoral research fellow with the Black Dog Institute and School of Psychiatry, University of New South Wales (Black Dog Institute, University of New South Wales, The Villa, Prince of Wales Hospital, Randwick NSW 2031, Australia. E-mail: k.baikie@unsw.edu.au). She completed her PhD in the use of expressive writing in the Department of Psychology, Macquarie University, Sydney. Her interests are in the application of expressive writing for different clinical populations and in working therapeutically with survivors of trauma, as well as general adult clinical psychology. Kay Wilhelm is a consultant psychiatrist in consultation liaison psychiatry at St Vincent's Hospital, Sydney, and the Mood Disorders Unit, Black Dog Institute, where she is also project leader for the General Practitioner Education Program. She is a clinical Associate Professor at the University of New South Wales. She has a long-standing interest in depression, especially gender issues and psychosocial risk factors, brief psychotherapy for depression and self-harm, as well as primary care and general hospital psychiatry. K.B. is supported by National Health and Medical Research Council Program Grant 222708. 
although more recently writing has been done at home or in a clinical setting. Participants often reveal a considerable range and depth of emotional trauma in their writing. Although many report being upset by the writing experience, they also find it valuable and meaningful (Pennebaker, 1997b). Control participants are asked to write as objectively and factually as possible about neutral topics such as a particular room or their plans for the day, without revealing their emotions or opinions. No feedback is given on the writing.

Owing to the nature of $A P T$, study results discussed below are not exhaustively referenced; instead, we have tried to give the most representative or comprehensive publications. For further reading on expressive writing and its implementation we recommend Lepore \& Smyth (2002), Pennebaker $(1997 a, b)$ and Sloan \& Marx (2004b).

\section{Immediate and longer-term effects of expressive writing}

The immediate impact of expressive writing is usually a short-term increase in distress, negative mood and physical symptoms, and a decrease in positive mood compared with controls. Expressive writing participants also rate their writing as significantly more personal, meaningful and emotional. However, at longer-term follow-up, many studies have continued to find evidence of health benefits in terms of objectively assessed outcomes, selfreported physical health outcomes and self-reported emotional health outcomes (Box 2).

\section{Objectively assessed outcomes}

Expressive writing results in significant improvements in longer-term physical health outcomes such as illness-related visits to the doctor (Pennebaker \& Beall, 1986; Pennebaker et al, 1988; Pennebaker \& Francis, 1996; King \& Miner, 2000), blood pressure (Davidson et al, 2002, citing Crow et al), lung function (Smyth et al, 1999), liver function (Francis \& Pennebaker, 1992) and number of days in hospital (Norman et al, 2004). Expressive writing has also produced significant benefits in a number of measures of immune system functioning (Pennebaker et al, 1988; Esterling et al, 1994; Booth et al, 1997; Petrie et al, 1995, 2004).

Significant benefits have also been found for such objective outcomes as students' grade point average (Pennebaker \& Francis, 1996; Cameron \& Nicholls, 1998), absenteeism from work (Francis \& Pennebaker, 1992), re-employment after job loss (Spera et al, 1994), working memory (Klein \& Boals, 2001) and sporting performance (Scott et al, 2003). In addition, writing
Box 2 Longer-term benefits of expressive writing

Health outcomes

- Fewer stress-related visits to the doctor

- Improved immune system functioning

- Reduced blood pressure

- Improved lung function

- Improved liver function

- Fewer days in hospital

- Improved mood/affect

- Feeling of greater psychological well-being

- Reduced depressive symptoms before examinations

- Fewer post-traumatic intrusion and avoidance symptoms

Social and behavioural outcomes

- Reduced absenteeism from work

- Quicker re-employment after job loss

- Improved working memory

- Improved sporting performance

- Higher students' grade point average

- Altered social and linguistic behaviour

about emotional topics changed the way that participants interacted with others, suggesting that writing may also have an impact on objectively assessed social and linguistic behaviour (Pennebaker \& Graybeal, 2001).

\section{Self-reported physical health outcomes}

Expressive writing also produces longer-term benefits in self-reported health outcomes such as visits to the doctor (Cameron \& Nicholls, 1998), physical symptoms (Park \& Blumberg, 2002) and number of days out of role because of illness (Pennebaker \& Beall, 1986; Smyth et al, 2001).

In general, expressive writing does not affect health-related behaviours such as exercise, diet or drug/alcohol use (Pennebaker et al, 1988).

\section{Self-reported emotional health outcomes}

Some studies have also found longer-term benefits of expressive writing for emotional health outcomes, including mood/affect (Pennebaker et al, 1988; Páez et al, 1999), psychological well-being (Park \& Blumberg, 2002), depressive symptoms before examinations (Lepore, 1997) and post-traumatic intrusion and avoidance symptoms (Klein \& Boals, 2001). However, the findings for emotional health are not as robust or as consistent as those for physical health. 


\section{Meta-analyses}

A meta-analysis of 13 studies using expressive writing with healthy participants (Smyth, 1998) found a significant overall benefit $(d=0.47$, $P<0.0001)$ and specific benefits in objective or selfreported physical health, psychological well-being, physiological functioning and general functioning outcomes. Smyth's review suggests that, for physically and psychologically healthy individuals, the effects produced by expressive writing are substantial and similar in magnitude to the effects of other psychological interventions, many of which are more involved, time-consuming and expensive.

In clinical populations, a meta-analysis (Frisina et al, 2004) of nine expressive writing studies also found a significant benefit for health $(d=0.19$, $P<0.05)$, although when analysed separately the effects for physical health outcomes in medically ill populations were significant $(d=0.21, P=0.01)$ but those for psychological health outcomes in psychiatric populations were not $(d=0.07, P=0.17)$. Although the benefits are more modest than in studies with healthy participants, this meta-analysis suggests that expressive writing nevertheless has positive effects in clinical populations.

\section{Who can benefit?}

\section{Medical conditions}

In comparisons with controls, expressive writing produced significant benefits for individuals with a variety of medical problems (Box 3). Study participants with asthma or rheumatoid arthritis showed improvements in lung function and physician-rated disease severity respectively, following a laboratorybased writing progamme (Smyth et al, 1999), although people with rheumatoid arthritis using a home-based videotaped programme showed no benefit (Broderick et al, 2004). Some studies found

Box 3 Medical conditions that might benefit from expressive writing programmes

- Lung functioning in asthma

- Disease severity in rheumatoid arthritis

- Pain and physical health in cancer

- Immune response in HIV infection

- Hospitalisations for cystic fibrosis

- Pain intensity in women with chronic pelvic pain

- Sleep-onset latency in poor sleepers

- Post-operative course that patients with cancer reported benefits such as better physical health, reduced pain and reduced need to use healthcare services (Rosenberg et al, 2002; Stanton \& Danoff-Burg, 2002), although others failed to find any benefits (Walker et al, 1999; de Moor et al, 2002). Patients with HIV infection showed improved immune response similar to that seen in monotherapy with anti-HIV drugs (Petrie et al, 2004) and individuals with cystic fibrosis showed a significant reduction in hospital-days over a 3-month period (Taylor et al, 2003). Women with chronic pelvic pain reported reductions in pain intensity ratings (Norman et al, 2004) and poor sleepers reported shorter sleep-onset latency (Harvey \& Farrell, 2003). Benefits have also been found for post-operative course after papilloma resection (Solano et al, 2003) and for primary care patients (Klapow et al, 2001; Gidron et al, 2002).

\section{Psychological conditions}

Other studies have investigated expressive writing in preselected groups of trauma survivors and individuals with specific psychological difficulties, with mixed results. Students with a trauma history have shown improvements in physical health (Greenberg et al, 1996; Sloan \& Marx, 2004a), posttraumatic stress disorder (PTSD) symptomatology and other aspects of psychological health (Schoutrop et al, 1997, 2002; Sloan \& Marx, 2004a), although not all studies find benefits (Deters \& Range, 2003).

Limited benefits were obtained for male psychiatric prison inmates (Richards et al, 2000), victims of natural disaster (Smyth et al, 2002) and individuals who had experienced a recent relationship breakup (Lepore \& Greenberg, 2002).

Expressive writing was beneficial, but not significantly more so than control writing, for females writing about body image (Earnhardt et al, 2002), children of alcoholics (Gallant \& Lafreniere, 2003), caregivers of children with chronic illness (Schwartz \& Drotar, 2004), students screened for suicidality (Kovac \& Range, 2002) and individuals who had experienced a bereavement (Range et al, 2000; O'Connor et al, 2003).

Compared with controls, expressive writing was detrimental for adult survivors of childhood abuse (Batten et al, 2002) and for a small sample of eight Vietnam veterans with PTSD (Gidron et al, 1996).

Our review of the literature shows that psychological health benefits tend to be more often found when participants' traumas and/or symptoms are clinically more severe, although results are inconsistent. One explanation for this inconsistency may be that many of the studies with null findings instructed participants to write about the specific traumatic event they were selected for, rather than 
using the standard instructions (Box 1), which allow them to write about events of their choosing. In studies where expressive writing was beneficial, many participants wrote about topics other than their particular physical illness or psychological problem, but still showed improvements in that area (Smyth \& Pennebaker, 1999).

\section{Individual differences}

In addition to studying specific health populations, researchers have explored various individual difference indices to identify those subgroups for whom expressive writing is most beneficial. Results have been inconsistent. Variables generally found to be unrelated to outcome include age, trauma severity, baseline physical and psychological health levels, negative affectivity and measures of inhibition and prior disclosure.

Smyth's (1998) meta-analysis found that the effects were greater for males than for females. Expressive writing is more beneficial for those high in alexithymia (Páez et al, 1999; Baikie, 2003; Solano et al, 2003) and high in splitting (Baikie, 2003), characteristics often seen in patients with psychosomatic disorders and borderline personality disorder respectively, suggesting potential for the use of expressive writing in these populations.

\section{Conclusion}

Overall, studies examining expressive writing demonstrate some beneficial effects in physical and/ or psychological health. Although the empirical findings are at times equivocal and further research is required to clarify populations for whom writing is clearly effective, there is sufficient evidence for clinicians to begin applying expressive writing in therapeutic settings with caution. Indeed, Spiegel (1999) noted that a drug intervention reporting medium effect sizes similar to those found for expressive writing (Smyth, 1998) would be regarded as a major medical advance.

\section{How does it work?}

Although the exact mechanism by which expressive writing confers health benefits is still unclear, there have been a number of potential explanations (Box 4) (see also Sloan \& Marx, 2004b).

\section{Emotional catharsis}

There is little support for the initial hypothesis that expressive writing operates through a process of
Box 4 Mechanisms by which expressive writing might work

- Emotional catharsis: Unlikely

- Confronting previously inhibited emotions: May reduce physiological stress resulting from inhibition, but unlikely to be the only explanation

- Cognitive processing: It is likely that the development of a coherent narrative helps to reorganise and structure traumatic memories, resulting in more adaptive internal schemas

- Repeated exposure: May involve extinction of negative emotional responses to traumatic memories, but some equivocal findings

emotional catharsis or venting of negative feelings. Writing only about the emotions associated with a trauma is not as beneficial as writing about both the event and the emotions (Pennebaker \& Beall, 1986). Furthermore, expressive writing results in immediate increase in negative affect rather than immediate relief of emotional tension, and the obtained health benefits are unrelated to the amount of negative emotion or distress either expressed or reported just after writing (Smyth, 1998).

\section{Emotional inhibition and confrontation}

Pennebaker's (1985) theory proposed that actively inhibiting thoughts and feelings about traumatic events requires effort, serves as a cumulative stressor on the body and is associated with increased physiological activity, obsessive thinking or ruminating about the event, and longer-term disease. Confronting a trauma through talking or writing about it and acknowledging the associated emotions is thought to reduce the physiological work of inhibition, gradually lowering the overall stress on the body. Such confrontation involves translating the event into words, enabling cognitive integration and understanding of it, which further contribute to the reduction in physiological activity associated with inhibition and ruminations (Pennebaker, 1985).

This theory has intuitive appeal but mixed empirical support. Studies have shown that expressive writing results in significant improvements in various biochemical markers of physical and immune functioning (Pennebaker et al, 1988; Esterling et al, 1994; Petrie et al, 1995; Booth et al, 1997). This suggests that written disclosure may reduce the physiological stress on the body caused by inhibition, although it does not necessarily mean that disinhibition is the causal mechanism underlying 
these biological effects. On the other hand, participants writing about previously undisclosed traumas showed no differences in health outcomes from those writing about previously disclosed traumas (Greenberg \& Stone, 1992) and participants writing about imaginary traumas that they had not actually experienced, and therefore could not have inhibited, also demonstrated significant improvements in physical health (Greenberg et al, 1996). Therefore, although inhibition may play a part, the observed benefits of writing are not entirely due to reductions in inhibition.

\section{Development of a coherent narrative}

A computerised text analysis system, Linguistic Inquiry and Word Count (LIWC; Pennebaker et al, 2001), was specifically designed to determine whether certain linguistic markers might be associated with improvements in health. The LIWC program analyses the writing tasks by calculating the percentage of words in the text matching each of 82 predefined language categories. The most consistent finding has been that, over the course of writing, participants whose health improved used more positive-emotion words, a moderate number of negative-emotion words and an increased number of 'cognitive mechanism' words (the latter include insight words such as understand, realise and causal words such as because, reason) (Pennebaker, 1997b).

The subsequent suggestion that the beneficial effect of expressive writing is the development of a coherent narrative over time, reflecting increasing cognitive processing of the experience, is consistent with the literature on traumatic memory and trauma treatment (e.g. van der Kolk et al, 1996). In addition, recent linguistic studies have shown that session-tosession variations in pronoun use are related to health improvements, which may reflect a transformation in the way people think about themselves in relation to others and the world (Pennebaker, 2002).

\section{Cognitive processing}

More direct investigations of cognitive processing as a potential mechanism suggest that writing may help the writer to organise and structure the traumatic memory, resulting in more adaptive, integrated schemas about self, others and the world (Harber \& Pennebaker, 1992).

Although the cognitive processing hypothesis has been difficult to evaluate empirically owing to the difficulty of measuring cognitive changes, there is evidence that narrative formation and coherence are necessary for expressive writing to be beneficial (Smyth et al, 2001) and that expressive writing increases working memory capacity, which may reflect improved cognitive processing (Klein \& Boals, 2001).

\section{Exposure}

The effectiveness of prolonged exposure as a treatment for post-traumatic stress (Foa \& Rothbaum, 1998) led to the suggestion that the writing paradigm may produce extinction of negative emotional responses through repeated writing about traumatic memories (Lepore et al, 2002). This hypothesis has met with mixed support. There is some evidence that exposure may underlie the emotional health benefits of expressive writing (Sloan \& Marx, 2004a; Sloan et $a l, 2005)$. However, many participants benefit from writing regardless of whether they write about the same traumatic experience or different experiences at each writing session. Furthermore, writing sessions are usually considerably shorter than the 45-90 minutes deemed necessary to facilitate emotional habituation. Some of the benefits of expressive writing may be a result of repeated exposure to negative emotional experiences.

\section{Conclusion}

As can be seen, each of the proposed theories has supporting and contradictory evidence (Sloan \& Marx, 2004b). The mechanism of action appears to be complex, with the demonstrated benefits potentially resulting from some combination of immediate cognitive and/or emotional changes, longer-term cognitive and/or emotional changes, social processes and biological effects, rather than being accounted for by any single factor (Pennebaker, 2004).

\section{How to use expressive writing as a therapeutic tool}

Expressive writing has primarily been investigated in carefully controlled research settings, with results generalising well across laboratories. However, given its simplicity, expressive writing appears to have great potential as a therapeutic tool in diverse clinical settings or as a means of self-help, either alone or as an adjunct to traditional therapies (see also Smyth \& Helm, 2003; Pennebaker, 2004). For example, promising results have been found using e-mailbased writing assignments (Sheese et al, 2004), an internet-based writing intervention for posttraumatic stress (Lange et al, 2000) and writing tasks for couples recovering from an extramarital affair (Snyder et al, 2004). 


\section{Box 5 Suggestions for the clinical use of} expressive writing

- Expressive writing tasks can be set as homework, or can be carried out before, during or after a session

- Writing should be carried out in a private, personalised place, free from distractions

- Write on three or four occasions, usually on consecutive days or weeks

- Set aside 30 minutes, with 20 minutes for writing and 10 minutes for patients to compose themselves afterwards

- Use the writing instructions shown in Box 1

- Let the patient select a traumatic/stressful experience: do not specify a particular trauma or event

- Allow the patient to structure the writing rather than imposing structure

- If possible, give the patient the option to write by hand or on a computer

- Explain to the patients that their writing is private, for themselves not for you and that confidentiality and anonymity are assured; explain that you will not read their writing unless they want you to

- Do not give feedback

- Writing should be kept by the patient or separate from the clinical file

In extending the paradigm to clinical settings, following as much of the traditional protocol as possible will make it more likely that health benefits will be achieved (see also Batten, 2002). Although there is no direct evidence, it has been suggested that the more structured approach of the expressive writing paradigm is more beneficial than simple diary-keeping (Smyth \& Pennebaker, 1999). In addition, it seems that incorporating both the cognitive and the emotional components of the experience (i.e. thoughts and feelings) is helpful (Pennebaker \& Beall, 1986; Smyth \& Pennebaker, 1999). Suggestions for using expressive writing in clinical or self-help settings are given in Box 5.

As the application of expressive writing outside of research settings is relatively recent, it is recommended that clinicians collect some data to assess its effectiveness in their particular setting, including appropriate pre- and post-writing measures of physical health, psychological health or general functioning (see Box 6).

\section{Box 6 Notes on expressive writing}

As a clinical psychiatrist working in a public teaching hospital, I (K.W.) have found expressive writing to be a useful addition to my repertoire of short-term psychological interventions for people who harm themselves, in the medical wards and for out-patients with stress-related symptoms, anxiety and depression. I use it together with daily mood charts, problemsolving, goal-setting, relaxation, mindfulness, exercise prescription and other interventions that form part of the Black Dog Institute's general practitioner education programme (for related screening measures and information sheets follow the prompt for Clinician aids on the Institute's website at http://www. blackdoginstitute.org.au ).

I keep a series of empty journals of different colours (to offer a choice) and ask patients to write on four occasions, following the instructions in Box 1. They are told to write for themselves and it is up to them whether they want to share the writing with anyone else. Later, I give some feedback on the changes in writing (after linguistic analysis), if requested.

Writing has helped people to resolve longstanding issues about relationships at home and work, and to put into words feelings that have been too sensitive to describe face to face. Some patients have shown their writing to significant others and found this helpful.

Research suggests that writing may be more beneficial for men and, in my experience, men have certainly found it an acceptable intervention.

The writing is intended for patients to use as a short-term intervention to start a process of dialogue with themselves or to 'unblock' a difficult issue. It is not intended to replace faceto-face interaction and is best done with a follow-up appointment for debriefing.

As the whole point is to bring up issues that are emotionally charged, it is important to work out the best timing for the writing and to have a contingency plan if the patient becomes distressed.

For some people the experience has been extremely helpful and has quickly resolved issues that have been mulled over-sometimes for years - with no resolution.

I encourage people to continue to use their journals in whatever way they think best, and most do so. 


\section{Cautions and limitations}

Expressive writing is generally associated with an immediate increase in negative affect, but this shortterm distress does not appear to be detrimental or to pose a longer-term risk to participants (Hockemeyer et al, 1999). Given the large number of studies conducted to date, with only a few finding any worsening of symptoms for those writing about traumatic experiences, the expressive writing paradigm appears to be reasonably safe for participants, even if no specific benefits are obtained. However, it is recommended that patients be told that they can stop writing at any time, should they wish, and appropriate contact numbers should be made available in case of distress. Patients should be encouraged to write for a maximum of $20 \mathrm{~min}$ at each session, so that the task does not seem too overwhelming, although they may choose to continue writing once the time is up if they wish and if this is feasible.

Regardless of the demonstrated benefits of expressive writing, it should not replace appropriate medical or psychological treatment in clinical populations; it should be used as an adjunct to standard treatment while further research is being conducted.

\section{References}

Baikie, K. A. (2003) Rewriting Trauma: How and for Whom Does the Writing Paradigm Work? Doctoral dissertation Sydney: Macquarie University.

Batten, S. V. (2002) Written disclosure as a therapeutic tool. In Innovations in Clinical Practice: A Source Book (vol. 20) (eds L. VandeCreek \& T. L. Jackson), pp. 257-268. Sarasota, FL: Professional Resource Press.

Batten, S. V., Follette, V. M., Rasmussen Hall, M. L., et al (2002) Physical and psychological effects of written disclosure among sexual abuse survivors. Behavior Therapy, 33, 107-122.

Booth, R. J., Petrie, K. J., \& Pennebaker, J. W. (1997) Changes in circulating lymphocyte numbers following emotional disclosure: evidence of buffering? Stress Medicine, 13, 2329

Broderick, J. E., Stone, A. A., Smyth, J. M., et al (2004) The feasibility and effectiveness of an expressive writing intervention for rheumatoid arthritis via home-based videotaped instructions. Annals of Behavioral Medicine, 27, $50-59$.

Cameron, L. D., \& Nicholls, G. (1998) Expression of stressful experiences through writing: Effects of a self-regulation manipulation for pessimists and optimists. Health Psychology, 17, 84-92.

Davidson, K., Schwartz, A. R., Sheffield, D., et al (2002) Expressive writing and blood pressure. In The Writing Cure: How Expressive Writing Promotes Health and Emotional Well-being (eds S. J. Lepore \& J. M. Smyth), pp. 17-30. Washington, DC: American Psychological Association.

de Moor, C., Sterner, J., Hall, M., et al (2002) A pilot study of the effects of expressive writing on psychological and behavioral adjustment in patients enrolled in a Phase II trial of vaccine therapy for metastatic renal cell carcinoma. Health Psychology, 21, 615-619.
Deters, P. B. \& Range, L. M. (2003) Does writing reduce posttraumatic stress disorder symptoms? Violence and Victims, 18, 569-580.

Earnhardt, J. L., Martz, D. M., Ballard, M. E., et al (2002) A writing intervention for negative body image: Pennebaker fails to surpass the placebo. Journal of College Student Psychotherapy, 17, 19-35.

Esterling, B. A., Antoni, M. H., Fletcher, M. A., et al (1994) Emotional disclosure through writing or speaking modulates latent Epstein-Barr virus antibody titers. Journal of Consulting and Clinical Psychology, 62, 130-140.

Foa, E. B. \& Rothbaum, B. O. (1998) Treating the Trauma of Rape: Cognitive-Behavioral Therapy for PTSD. New York: Guilford Press.

Francis, M. E., \& Pennebaker, J. W. (1992) Putting stress into words. The impact of writing on physiological, absentee, and self-reported emotional well-being measures. American Journal of Health Promotion, 6, 280-287.

Frisina, P. G., Borod, J. C. \& Lepore, S. J. (2004) A metaanalysis of the effects of written emotional disclosure on the health outcomes of clinical populations. Journal of Nervous and Mental Disease, 192, 629-634.

Gallant, M. D., \& Lafreniere, K. D. (2003) Effects of an emotional disclosure writing task on the physical and psychological functioning of children of alcoholics. Alcoholism Treatment Quarterly, 21, 55-66.

Gidron, Y., Peri, T., Connolly, J. F., et al (1996) Written disclosure in posttraumatic stress disorder: is it beneficial for the patient? Journal of Nervous and Mental Disease, 184, 505-507.

Gidron, Y., Duncan, E., Lazar, A., et al (2002) Effects of guided written disclosure of stressful experiences on clinic visits and symptoms in frequent clinic attenders. Family Practice, 19, 161-166.

Greenberg, M. A. \& Stone, A. A. (1992) Emotional disclosure about traumas and its relation to health. Effects of previous disclosure and trauma severity. Journal of Personality and Social Psychology, 63, 75-84.

Greenberg, M. A., Wortman, C. B. \& Stone, A. A. (1996) Emotional expression and physical heath. Revising traumatic memories or fostering self-regulation? Journal of Personality and Social Psychology, 71, 588-602.

Harber, K. D., \& Pennebaker, J. W. (1992) Overcoming traumatic memories. In The Handbook of Emotion and Memory: Research and Theory (ed. S.-Å. Christianson), pp. 359-387. Hillsdale, NJ: Lawrence Erlbaum Associates.

Harvey, A. G. \& Farrell, C. (2003) The efficacy of a Pennebakerlike writing intervention for poor sleepers. Behavioral Sleep Medicine, 1, 115-124.

Hockemeyer, J. R., Smyth, J. M., Anderson, C. F., et al (1999) Is it safe to write? Evaluating the short-term distress produced by writing about emotionally traumatic experiences [abstract]. Psychosomatic Medicine, 61, 99.

King, L. A. \& Miner, K. N. (2000) Writing about the perceived benefits of traumatic events: implications for physical health. Personality and Social Psychology Bulletin, 26, 220230.

Klapow, J. C., Schmidt, S. M., Taylor, L. A., et al (2001) Symptom management in older primary care patients. Feasibility of an experimental, written self-disclosure protocol. Annals of Internal Medicine, 134 (9 Pt 2), 905-911.

Klein, K. \& Boals, A. (2001) Expressive writing can increase working memory capacity. Journal of Experimental Psychology: General, 130, 520-533.

Kovac, S. H. \& Range, L. M. (2002) Does writing about suicidal thoughts and feelings reduce them? Suicide and Life-Threatening Behavior, 32, 428-440.

Lange, A., van de Ven, J. P., Schrieken, B. A., et al (2000) Internet-mediated, protocol-driven treatment of psychological dysfunction. Journal of Telemedicine and Telecare, 6, $15-21$

Lepore, S. J. (1997) Expressive writing moderates the relation between intrusive thoughts and depressive symptoms. Journal of Personality and Social Psychology, 73, 1030-1037.

Lepore, S. J. \& Greenberg, M. A. (2002) Mending broken hearts: Effects of expressive writing on mood, cognitive 
processing, social adjustment and health following a relationship breakup. Psychology and Health, 17, 547-560.

Lepore, S. J. \& Smyth, J. M. (eds) (2002) The Writing Cure: How Expressive Writing Promotes Health and Emotional Well-being. Washington, DC: American Psychological Association.

Lepore, S. J., Greenberg, M. A., Bruno, M., et al (2002) Expressive writing and health: self-regulation of emotionrelated experience, physiology, and behavior. In The Writing Cure: How Expressive Writing Promotes Health and Emotional Well-being (eds S. J. Lepore \& J. M. Smyth), pp. 99-117) Washington, DC: American Psychological Association.

Norman, S. A., Lumley, M. A., Dooley, J. A., et al (2004) For whom does it work? Moderators of the effects of written emotional disclosure in a randomized trial among women with chronic pelvic pain. Psychosomatic Medicine, 66, 174183

O'Connor, M., Nikoletti, S., Kristjanson, L. J., et al (2003) Writing therapy for the bereaved. Evaluation of an intervention. Journal of Palliative Medicine, 6, 195-204.

Páez, D., Velasco, C. \& Gonzalez, J. L. (1999) Expressive writing and the role of alexythimia as a dispositional deficit in self-disclosure and psychological health. Journal of Personality and Social Psychology, 77, 630-641.

Park, C. L. \& Blumberg, C. J. (2002) Disclosing trauma through writing: testing the meaning-making hypothesis. Cognitive Therapy and Research, 26, 597-616.

Pennebaker, J. W. (1985) Traumatic experience and psychosomatic disease. Exploring the roles of behavioural inhibition, obsession, and confiding. Canadian Psychology, 26, 82-95.

Pennebaker, J. W. (1994) Some Suggestions for Running a Confession Study. http://homepage.psy.utexas.edu/ homepage/faculty / Pennebaker/Reprints / Hints.DOC

Pennebaker, J. W. (1997a) Opening Up: The Healing Power of Expressing Emotions. New York: Guilford Press.

Pennebaker, J. W. (1997b) Writing about emotional experiences as a therapeutic process. Psychological Science, 8, 162-166.

Pennebaker, J. W. (2002) What our words can say about us. Toward a broader language psychology. Psychological Science Agenda, 15, 8-9.

Pennebaker, J. W. (2004) Theories, therapies, and taxpayers. On the complexities of the expressive writing paradigm. Clinical Psychology: Science and Practice, 11, 138-142.

Pennebaker, J. W. \& Beall, S. K. (1986) Confronting a traumatic event. Toward an understanding of inhibition and disease. Journal of Abnormal Psychology, 95, 274-281.

Pennebaker, J. W. \& Francis, M. E. (1996) Cognitive, emotional, and language processes in disclosure. Cognition and Emotion, 10, 601-626.

Pennebaker, J. W. \& Graybeal, A. (2001) Patterns of natural language use. Disclosure, personality, and social integration. Current Directions, 10, 90-93.

Pennebaker, J. W., Kiecolt-Glaser, J. K. \& Glaser, R. (1988) Disclosure of traumas and immune function. Health implications for psychotherapy. Journal of Consulting and Clinical Psychology, 56, 239-245.

Pennebaker, J. W., Francis, M. E. \& Booth, R. J. (2001) Linguistic Inquiry and Word Count (LIWC2001). Mahwah, NJ: Erlbaum.

Petrie, K. J., Booth, R. J., Pennebaker, J. W., et al (1995) Disclosure of trauma and immune response to a hepatitis B vaccination program. Journal of Consulting and Clinical Psychology, 63, 787-792.

Petrie, K. J., Fontanilla, I., Thomas, M. G., et al (2004) Effect of written emotional expression on immune function in patients with Human Immunodeficiency Virus infection. A randomized trial. Psychosomatic Medicine, 66, 272-275.

Range, L. M., Kovac, S. H. \& Marion, M. S. (2000) Does writing about the bereavement lessen grief following sudden, unintentional death? Death Studies, 24, 115-134.

Richards, J. M., Beal, W. E., Seagal, J. D., et al (2000) Effects of disclosure of traumatic events on illness behavior among psychiatric prison inmates. Journal of Abnormal Psychology, 109, 156-160.
Rosenberg, H. J., Rosenberg, S. D., Ernstoff, M. S., et al (2002) Expressive disclosure and health outcomes in a prostate cancer population. International Journal of Psychiatry in Medicine, 32, 37-53.

Schoutrop, M. J. A., Lange, A., Brosschot, J. F., et al (1997) Overcoming traumatic events by means of writing assignments. In The (Non)Expression of Emotions in Health and Disease (eds A. Vingerhoets, F. van Bussel \& J. Boelhower), pp. 279-289. Tilburg: Tilburg University Press.

Schoutrop, M. J. A., Lange, A., Hanewald, G., et al (2002) Structured writing and processing major stressful events. A controlled trial. Psychotherapy and Psychosomatics, 71, 151-157.

Schwartz, L. \& Drotar, D. (2004) Effects of written emotional disclosure on caregivers of children and adolescents with chronic illness. Journal of Pediatric Psychology, 29, 105-118.

Scott, V. B., Robare, R. D., Raines, D. B., et al (2003) Emotive writing moderates the relationship between mood awareness and athletic performance in collegiate tennis players. North American Journal of Psychology, 5, 311-324.

Sheese, B. E., Brown, E. L. \& Graziano, W. G. (2004) Emotional expression in cyberspace. Searching for moderators of the Pennebaker disclosure effect via e-mail. Health Psychology, 23, 457-464.

Sloan, D. M. \& Marx, B. P. (2004a) A closer examination of the structured written disclosure procedure. Journal of Consulting and Clinical Psychology, 72, 165-175.

Sloan, D. M. \& Marx, B. P. (2004b) Taking pen to hand. Evaluating theories underlying the written disclosure paradigm. Clinical Psychology: Science and Practice, 11, 121137.

Sloan, D. M., Marx, B. P. \& Epstein, E. M. (2005) Further examination of the exposure model underlying the efficacy of written emotional disclosure. Journal of Consulting and Clinical Psychology, 73, 549-554.

Smyth, J. M. (1998) Written emotional expression. Effect sizes, outcome types, and moderating variables. Journal of Consulting and Clinical Psychology, 66, 174-184.

Smyth, J. M. \& Helm, R. (2003) Focused expressive writing as self-help for stress and trauma. Journal of Clinical Psychology, 59, 227-235.

Smyth, J. M. \& Pennebaker, J. W. (1999) Sharing one's story: translating emotional experiences into words as a coping tool. In Coping: The Psychology of What Works (ed. C. R. Snyder), pp. 70-89. New York: Oxford University Press.

Smyth, J. M., Stone, A. A., Hurewitz, A., et al (1999) Effects of writing about stressful experiences on symptom reduction in patients with asthma or rheumatoid arthritis. A randomized trial. JAMA, 281, 1304-1309.

Smyth, J. M., True, N. \& Souto, J. (2001) Effects of writing about traumatic experiences. The necessity for narrative structuring. Journal of Social and Clinical Psychology, 20, 161-172.

Smyth, J. M., Hockemeyer, J. R., Anderson, C., et al (2002) Structured writing about a natural disaster buffers the effect of intrusive thoughts on negative affect and physical symptoms. Australasian Journal of Disaster and Traumatic Studies, 1. http://www.massey.ac.nz/\%7Etrauma/ issues / 2002-1/smyth.htm

Snyder, D. K., Gordon, K. \& Baucom, D. H. (2004) Treating affair couples. Extending the written disclosure paradigm to relationship trauma. Clinical Psychology: Science and Practice, 11, 155-159.

Solano, L., Donati, V., Pecci, F., et al (2003) Postoperative course after papilloma resection. Effects of written disclosure of the experience in subjects with different alexithymia levels. Psychosomatic Medicine, 65, 477-484.

Spera, S. P., Buhrfeind, E. D. \& Pennebaker, J. W. (1994) Expressive writing and coping with job loss. Academy of Management Journal, 37, 722-733.

Spiegel, D. (1999) Healing words. Emotional expression and disease outcome. JAMA, 281, 1328.

Stanton, A. L. \& Danoff-Burg, S. (2002) Emotional expression, expressive writing, and cancer. In The Writing 
Cure: How Expressive Writing Promotes Health and Emotional Well-being (eds S. J. Lepore \& J. M. Smyth), pp. 31-51. Washington, DC: American Psychological Association.

Taylor, L. A., Wallander, J. L., Anderson, D., et al (2003) Improving health care utilization, improving chronic disease utilization, health status, and adjustment in adolescents and young adults with cystic fibrosis. A preliminary report. Journal of Clinical Psychology in Medical Settings, 10, 9-16.

van der Kolk, B. A., McFarlane, A. C. \& Weisaeth, L. (eds) (1996) Traumatic Stress: The Effects of Overwhelming Experience on Mind, Body, and Society. New York: Guilford Press.

Walker, B. L., Nail, L. M. \& Croyle, R. T. (1999) Does emotional expression make a difference in reactions to breast cancer? Oncology Nursing Forum, 26, 1025-1032.

\section{MCQs}

1 Successful outcome in expressive writing requires:

a a correct statement of the facts

$\mathrm{b}$ revealing subconscious thought processes

c being able to discuss the writing with a significant other

d being able to write freely for one's self

e verbal expression of emotion while writing.

2 Expressive writing has been shown to lead to:

a significant improvement in lung function in asthma sufferers b improved immune response in HIV patients

c decreased admission rates in cystic fibrosis

d improvements in joint stiffness in rheumatoid arthritis

e improved immune response in glandular fever.

3 People engaging in expressive writing are instructed to:

a write by hand or on a computer

b always play soft music while writing

c answer a set of questions provided

d write freely without worrying about grammar

e think about a topic prior to the session.

4 Expressive writing results in:

a higher college grades for students

b more exercise taken per week

c higher rates of re-employment after redundancy

d fewer visits to the general practitioner or health centre

e better diet.

5 The instruction for an expressive writing task should include:

a nominating a particular time of day

b writing about 'deepest thoughts and feelings'

c writing about the same topic on each occasion

d writing about a very traumatic experience

e specific instruction not to write at bedtime. 